\title{
Échec de la mise en place de dents retenues et incluses
}

\author{
Marion Pignoly ${ }^{1 *}$, Virginie Monnet-Corti ${ }^{2}$, Michel Le Gall ${ }^{3}$ \\ 168 avenue de St Julien, 13012 Marseille, France \\ 2 Hôpital de la Timone-AP-HM, Pôle Odontologie, 264 rue Saint-Pierre, 13385 Marseille, France \\ 3314 boulevard Marcel Pagnol, Villa Gabrielle, 13400 Aubagne, France
}

MOTS CLÉS :

Dent incluse /

Dent retenue /

Thérapeutique

chirurgico-orthodontique

\begin{abstract}
RÉSUMÉ - La mise en place d'une dent incluse ou retenue, que ce soit chez l'enfant ou l'adulte, est un véritable défi pour le duo de praticiens que sont l'orthodontiste et le praticien en charge de la chirurgie de dégagement. La canine maxillaire est la dent la plus souvent incluse dans le secteur antérieur, avec une prévalence de $2 \%$ dans la population générale. On comprend donc le défi fonctionnel et esthétique de la thérapeutique chirurgico-orthodontique dont l'objectif sera de mettre en place la dent incluse ou retenue sur l'arcade. De nombreuses erreurs devront être évitées pour obtenir un résultat satisfaisant, tant pour le patient que pour son praticien. C'est en faisant un diagnostic précis de la localisation de la dent, grâce à des techniques d'imagerie modernes, que nous pourrons réaliser un acte chirurgical respectueux de la dent concernée et des structures anatomiques qui l'entourent. Les axes et les forces de tractions seront également pensés et réfléchis afin de déplacer la dent jusqu'à sa position finale sur l'arcade dans des conditions optimales. Le contexte parodontal sera optimisé et contrôlé à chaque étape de la prise en charge. En effet, le but étant d'aller au devant de l'apparition des défauts parodontaux, sachant qu'il est plus difficile de tenter leur réparation.
\end{abstract}

\begin{abstract}
Reason for failure in the treatment of impacted and retained teeth. The positioning of an impacted or retained tooth in either children or adults presents a real challenge for the two practitioners involved, the orthodontist and the surgeon in charge of the surgical exposure. The maxillary canine is the tooth most often impacted in the anterior segment, with a prevalence of $2 \%$ in the general population. One can understand, therefore, the functional and esthetic challenge for the surgical-orthodontic team whose aim will be to reposition the impacted or retained tooth in the arch. A large number of mistakes must be avoided in order to achieve a satisfactory result for both the patient and the practitioner. Modern imaging techniques should be used to obtain a precise diagnosis of the location of the tooth and to perform surgery which will spare the tooth in question as well as the surrounding anatomic structures. The directions and forces of the traction used will need to be carefully thought out in order to move the tooth into its final position on the arch in optimal conditions. The periodontal setting will be optimized and checked at every step of the treatment. The aim, in fact, is to anticipate the onset of periodontal problems on the grounds that prevention is easier than cure.
\end{abstract}

\section{Introduction}

La fréquence de l'inclusion des dents du secteur antérieur impose un diagnostic et une localisation précise de la dent afin qu'une thérapeutique

\footnotetext{
* Auteur pour correspondance : drmarionpignoly@hotmail.fr
}

chirurgico-orthodontique puisse être pensée et mise en œuvre. Ainsi, la dent incluse ou retenue peut être tractée jusqu'à sa position finale sur arcade sans danger pour elle, ni pour les dents adjacentes et les structures anatomiques environnantes. 


\section{Les échecs}

Une dent est dite incluse lorsqu'elle n'a pas fait son éruption après la date physiologique et que son sac folliculaire ne communique pas avec la cavité buccale. Elle se situe dans une crypte osseuse à un niveau plus ou moins profond du maxillaire inférieur ou supérieur. On considère qu'il y a inclusion en l'absence d'éruption 18 mois après la date habituelle, lorsque les apex radiculaires sont édifiés et fermés.

Une dent incluse se différencie d'une dent enclavée. Cette dernière n'a pas réalisé complètement son éruption mais communique avec la cavité buccale.

Enfin, une dent est dite retenue lorsqu'elle conserve un potentiel d'éruption (dent immature aux apex non édifiés). Des obstacles anatomiques, physiologiques ou pathologiques empêchent son éruption. Si cette dent retenue arrive à maturité, la rétention devient un enclavement ou une inclusion.

Après les troisièmes molaires, ce sont les dents du secteur antérieur qui sont le plus souvent incluses, avec notamment la canine maxillaire, incluse chez $2 \%$ de la population [6]. Cette inclusion est deux fois plus fréquente chez la femme [37] et la canine maxillaire est deux fois plus incluse que la canine mandibulaire [26].

Alors que de nombreuses études estiment que l'inclusion est palatine plutôt que vestibulaire dans $85 \%$ des cas [18], une étude plus récente utilisant des données obtenues grâce au cone-beam computerized tomography déclare que 45,2\% des canines maxillaires sont vestibulaires, 40,5\% sont palatines et $14,3 \%$ sont trans-alvéolaires [27].

\subsection{Prévalence des échecs}

Dans la littérature, les causes les plus fréquentes d'échec sont liées à des ancrages inadéquats (48,6 \%), des erreurs de localisation entraînant de mauvaises directions de traction $(40,5 \%)$, ou à des phénomènes d'ankylose (32,4\%) [4]. D’autres échecs, moins fréquents, peuvent être en lien avec des résorptions radiculaires des dents adjacentes à la dent incluse, des difficultés de traction dues à une position extrême de la dent à dégager ou encore à des décollements intempestifs d'ancrages. Ces échecs nécessitent la plupart du temps une révision du traitement qui aboutit à un succès dans la plupart des cas (71\%). Le recours à une nouvelle phase chirurgicale serait néanmoins nécessaire dans plus de $62 \%$ des cas, intervention dont le principal but est de changer la direction de traction.

\subsection{Différents types d'échecs}

\subsection{1. Échec de diagnostic ou de voie d'abord lié à l'imagerie}

Les examens radiologiques ont comme rôle de confirmer une suspicion clinique d'inclusion, et d'orienter la thérapeutique à adopter. Ils sont des aides précieuses à la préparation de l'acte chirurgical de dégagement de la dent incluse ou retenue.

\subsubsection{Apport de l'imagerie 3D}

Bien que certains clichés radiographiques en 2D, ou leur association, permettent de connaître la localisation de la dent incluse dans le sens antéropostérieur, il est de plus en plus fréquent de prescrire une imagerie en coupes, type scanner ou cone-beam computerized tomography (Fig. la-1c).

Cette imagerie 3D assure des reconstructions à l'échelle $1 / 1$, sans déformation, ni superposition et permet d'obtenir des informations fiables concernant :

- La localisation et l'orientation des germes retenus, dans les trois plans de l'espace, ou sous forme de reconstruction en volume, ainsi que leur rapport avec les structures anatomiques environnantes. Le scanner doit toujours comporter une reconstruction 3D [8] (Fig. 2).

- La localisation des obstacles à l'éruption et leur rapport avec la dent incluse.

- Les épaisseurs osseuses vestibulaire et palatine/linguale [30].

- Les résorptions éventuelles des dents adjacentes à la dent incluse ou de la dent incluse elle-même. En effet, dans le cas d'inclusion de canine maxillaire, il existe des résorptions radiculaires des incisives dans 20 à $30 \%$ des cas [27] (Fig. 3).

Le praticien chargé de la chirurgie a, grâce à cet examen, une idée précise de l'orientation de la dent incluse et peut envisager le positionnement de l'attache collée en fonction de l'orientation de la dent incluse et des forces de tractions à appliquer. À l'inverse, lorsque la radiographie 2D ne permet pas de déterminer avec précision la position vestibulo-palatine 


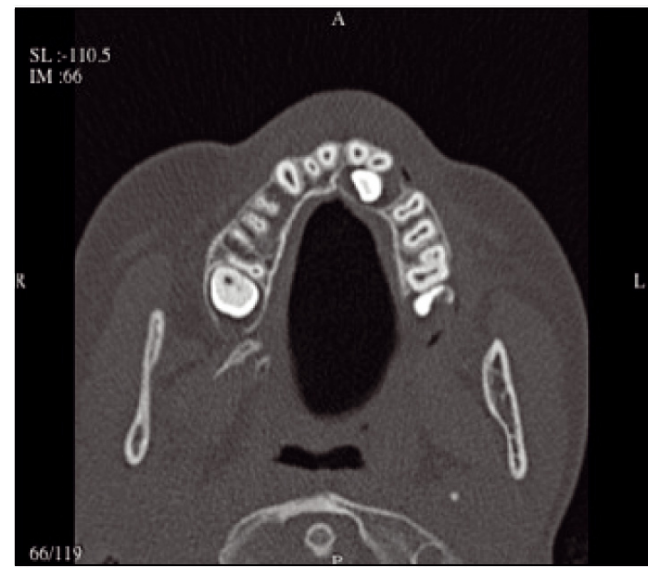

a

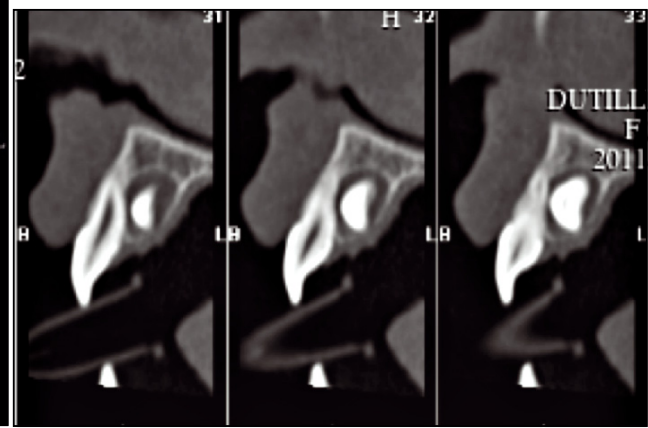

b

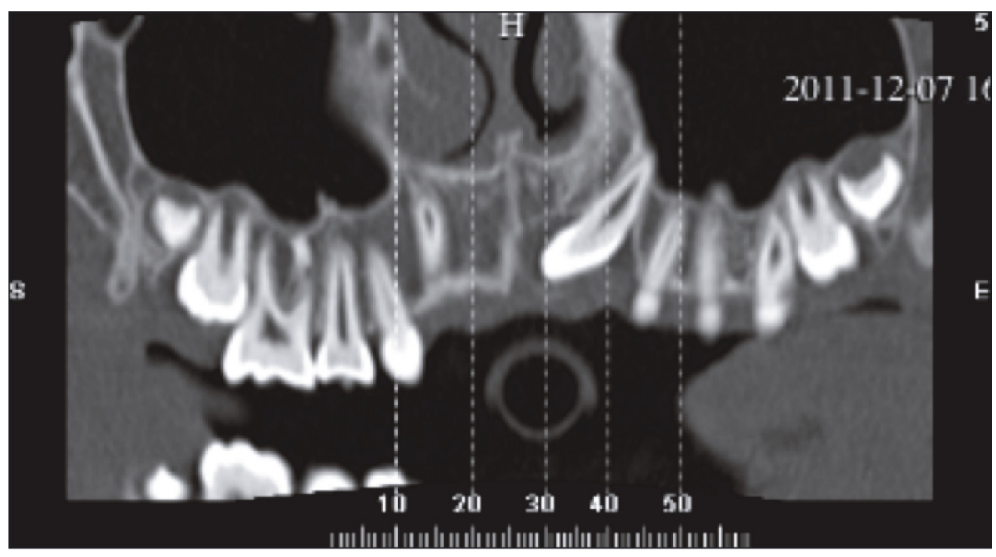

c

Figure 1

Coupes tomodensitométriques permettant de visualiser la dent incluse selon des coupes horizontales (a), sagittales (b) et frontales (c) à l'échelle 1/1.

de la dent incluse, il y a un risque que le praticienchirurgien aborde l'intervention dans une zone ne correspondant pas au site où se situe la dent incluse (Figs. 4 et 5). L'erreur initiale de localisation étant d'autant plus embêtante qu'elle a causé des séquelles, d'ordre parodontal ou dentaire, dans un secteur esthétique [4].

Certains logiciels permettent même de déterminer sur la scène 3D l'axe de traction optimal, ainsi que le point précis de collage de l'attache pour l'obtenir (Fig. 6). Un guide chirurgical est réalisé à partir de ces images et permet de transférer du virtuel au réel les informations sur la position de la dent incluse pendant la chirurgie [29].

Ainsi, bien qu'il reste un examen plus irradiant que les radiographies conventionnelles, et que son coût soit non négligeable, l'imagerie 3D est plus que jamais indiquée pour localiser précisément la dent incluse et les rapports intimes qu'elle peut avoir avec les structures adjacentes. En simulant l'intervention chirurgicale tout en anticipant le déplacement de la dent incluse, il permet de réaliser une chirurgie minimalement invasive, et éviter les lésions des dents et zones adjacentes.

\subsubsection{Possible mise en évidence de l'ankylose?}

Le phénomène d'ankylose correspond à un envahissement progressif de l'espace desmodontal par du tissu osseux et à la fusion anatomique anormale du cément de la dent atteinte à l'os alvéolaire sans interposition de tissu conjonctif.

Lankylose représente un véritable défi pour la prise en charge d'une dent incluse car le diagnostic est d'autant plus difficile à poser que la dent n'est pas sur arcade. De plus, une ankylose influe sur le pronostic de déplacement orthodontique de la dent, et oblige souvent le praticien à changer de thérapeutique en cours de traitement. 


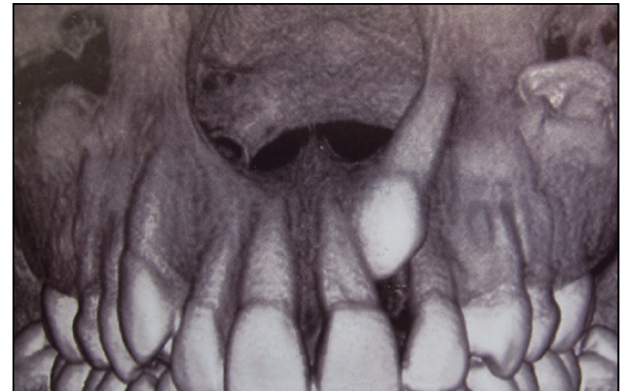

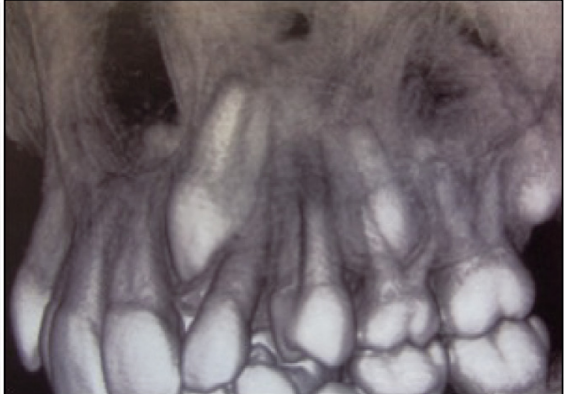

b

Figure 2

Images de reconstruction tridimensionnelle (d'après Korbendau, et al. [24]).

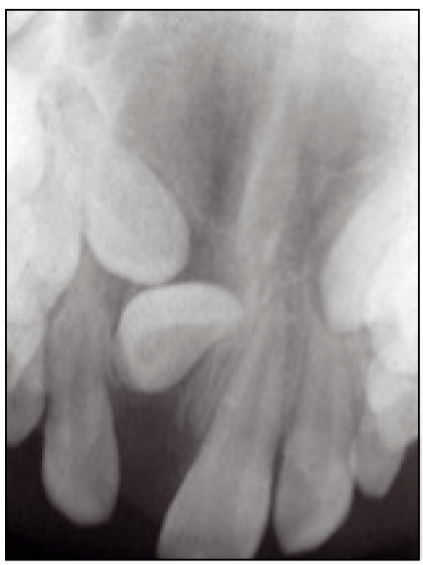

a

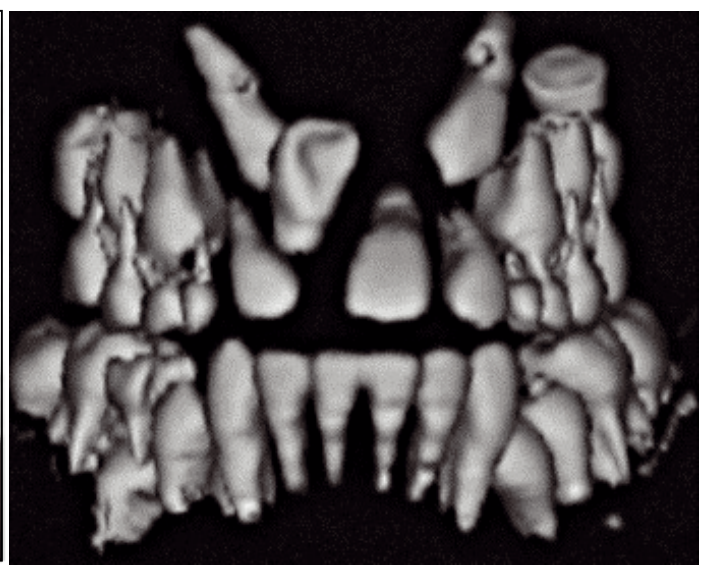

b

Figure 3

Alors que la radiographie 2D ne permet pas de diagnostiquer une résorption radiculaire de l'incisive centrale incluse chez un enfant de 9 ans, l'imagerie 3D, elle, permet de visualiser cette résorption (d'après Chen, et al. [12]).

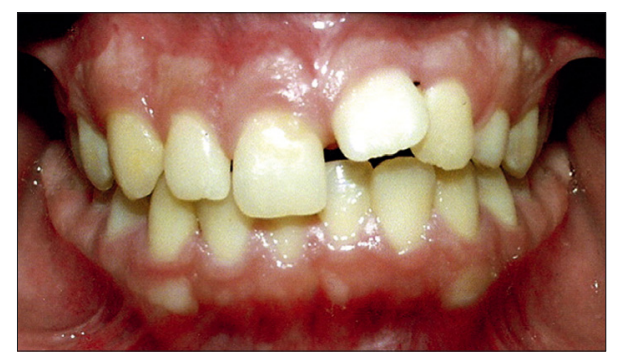

a

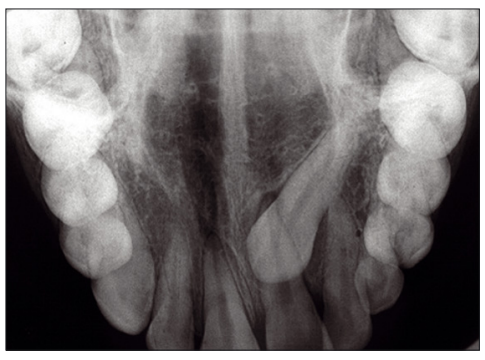

b

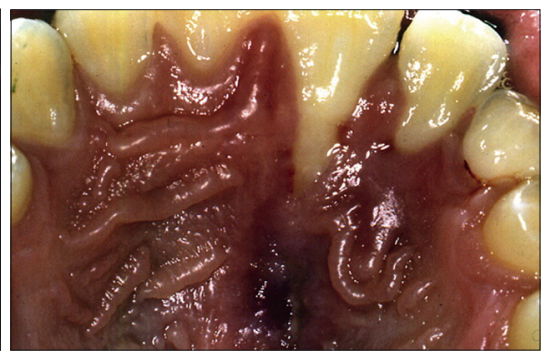

C

Figure 4

Récession palatine chez un patient dont la localisation de la canine maxillaire incluse a été diagnostiquée en palatin à l'aide d'imagerie 2D (d'après Becker, et al. [4]). 


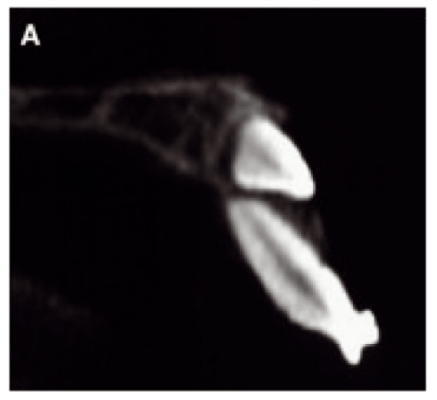

a

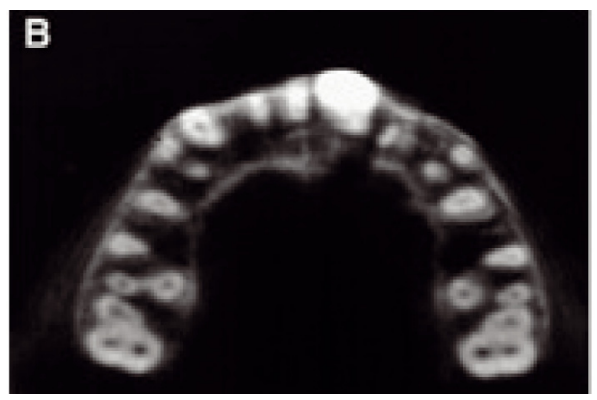

b

Figure 5

СBCT démontrant la position vestibulaire de la canine (d'après Becker, et al. [4]).

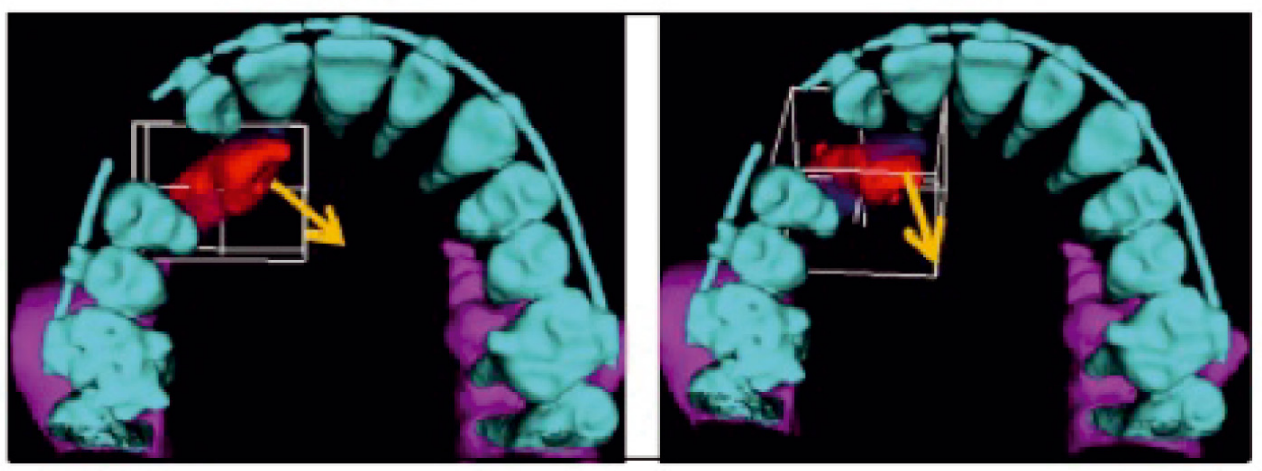

Figure 6

Simulation de la meilleure séquence de traction orthodontique (d'après Messaoudi, et al. [29]).

Lexamen 3D accompagné d'une analyse d'image et d'une reconstruction tridimensionnelle permet de révéler des lésions même de faible étendue et quelque soit la localisation (Fig. 7).

Limagerie 3D, seul examen pouvant révéler de façon fiable un phénomène d'ankylose au niveau d'une dent incluse, doit être systématiquement prescrit après trois mois d'échec de traction orthodontique sans autre raison apparente que l'ankylose [40].

\subsection{2. Échec lié à l'acte chirurgical}

Le praticien doit porter une attention particulière au contexte parodontal qu'il doit traiter. L'examen de la qualité du parodonte lui permet d'anticiper les risques et le résultat final d'un éventuel traitement chirurgico-orthodontique, notamment lors de dégagement chirurgical vestibulaire.

La localisation du point d'émergence de la dent retenue [23] joue un rôle clef dans le pronostic parodontal de la dent tractée sur arcade, et oriente la thérapeutique à adopter pour que l'environnement parodontal soit optimisé. En effet, de la position du point d'émergence dépend l'épaisseur des tissus de soutien, la morphologie et l'épaisseur des contours osseux et gingivaux. Elle est définie par sa situation rapportée à la ligne muco-gingivale. Le risque est que la dent émerge (spontanément ou suite à la traction orthodontique) au sein de la muqueuse alvéolaire et soit alors privée de gencive attachée. La chirurgie plastique parodontale pré-orthodontique permet d'optimiser un contexte parodontal de qualité.

\subsubsection{Incisions et positionnement du lambeau}

Le dégagement chirurgical d'une dent en dystopie vestibulaire peut être réalisé selon trois techniques distinctes : la gingivectomie, le lambeau repositionné ou les lambeaux déplacés apicalement et/ou latéralement.

Lindication de chacune de ces techniques est posée selon quatre critères [22] :

- La position verticale de la dent incluse par rapport à la ligne muco-gingivale. Si la dent est 


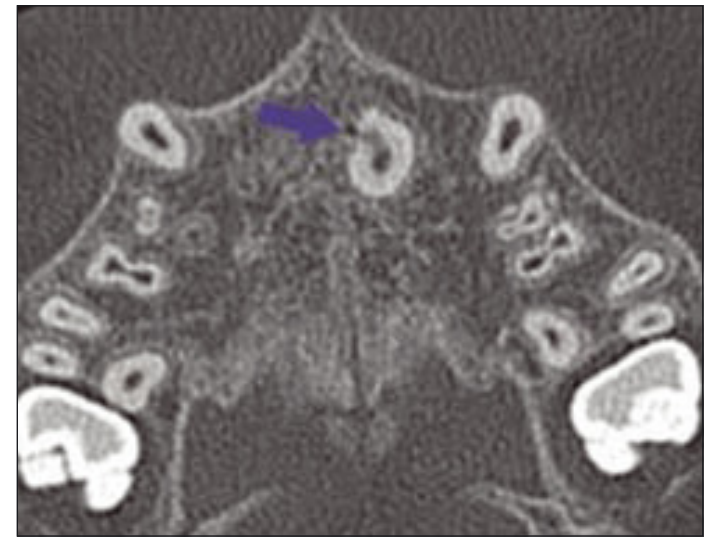

a

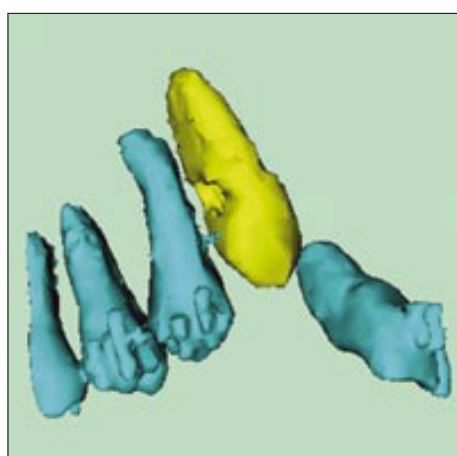

b

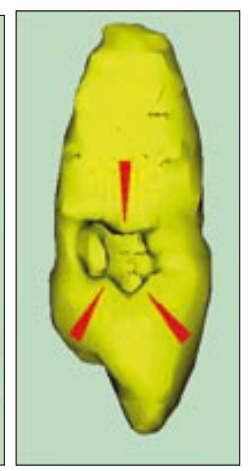

c

Figure 7

(a) Coupe tomodensitométrique révélant une zone d'ankylose au niveau d'une 21 incluse. (b et c) Reconstructions 3D permettant d'appréhender le volume d'ankylose et sa situation précise (d'après Paris, et al. [32]).

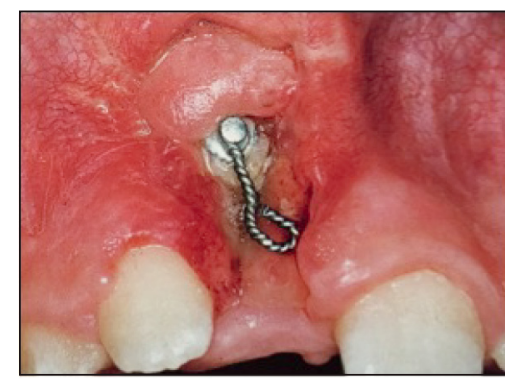

a

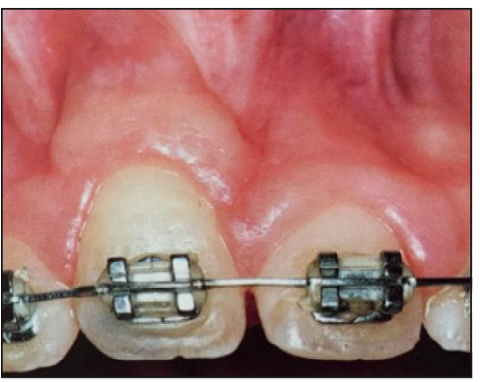

b

Figure 8

Résultat inesthétique du contour gingival après mise en place sur l'arcade de la 11 par un lambeau déplacé apicalement bien au-delà de la ligne muco-gingivale (d'après Korbendau, et al. [24]).

positionnée très apicalement à cette ligne, c'est le lambeau repositionné qui est indiqué. En effet, la gingivectomie induirait une récession vestibulaire importante et le lambeau déplacé apicalement engendrerait des risques de réintrusion et de récidive après le traitement orthodontique, ainsi qu'un mauvais rendu esthétique par déplacement trop apical du tissu kératinisé (Fig. 8).

De manière générale, le résultat est d'autant plus esthétique que le bord inférieur du lambeau est déplacé de façon raisonné et en-deça de la ligne muco-gingivale [24].

- La position vestibulaire ou trans-alvéolaire de la couronne de la dent incluse. Alors qu'une couronne positionnée en vestibulaire peut indiquer les trois techniques chirurgicales, une couronne positionnée en trans-alvéolaire doit indiquer le lambeau repositionné.

- La quantité de gencive kératinisée dans l'environnement de la dent incluse. Si elle est faible, le lambeau déplacé apicalement permet de conserver de la gencive kératinisée au collet de la dent incluse, contrairement à la gingivectomie. De plus, la largeur du lambeau à déplacer doit être au moins égale à la dimension mésio-distale de la couronne de la dent retenue.

- La position mésio-distale de la couronne de la dent incluse. C'est le lambeau déplacé latéralement qui est indiqué dans ce cas là. En effet, la gingivectomie mettrait en jeu le parodonte marginal des dents adjacentes.

De nombreux auteurs préconisent la technique du lambeau replacé. Les résultats esthétiques seraient systématiquement meilleurs avec cette technique qu'avec les lambeaux déplacés. Cependant, même si elle permet de recréer les conditions simulant une éruption physiologique, elle nécessite une traction « à l'aveugle» parfois difficile à appréhender. Lidéal pour se faire étant de faire passer le fil de traction 
par le tunnel créé après avulsion de la dent temporaire pour obtenir une émergence se rapprochant de l'éruption physiologique [13].

Pour ce qui est des dégagements de dents en dystopie palatine, l'environnement kératinisé de la zone palatine facilite la thérapeutique. Une attention doit tout de même être portée à l'environnement des dents adjacentes.

\subsubsection{Non respect de la quantité de tissu kératinisé}

Un dégagement chirurgical d'une dent retenue en position vestibulaire par gingivectomie ne peut être réalisé qu'en présence d'une hauteur suffisante de tissu kératinisé [9]. Celle-ci doit maintenir un bandeau de gencive kératinisée d'au moins $3 \mathrm{~mm}$ au collet de la dent après l'excision.

Si ce n'est pas le cas, la dent retenue en position vestibulaire est dégagée chirurgicalement par lambeau déplacé. Là encore, la quantité de tissu kératinisé déplacé doit correspondre, en hauteur et en épaisseur, à la quantité de tissu kératinisé mesurée au niveau de la dent contro-latérale [24].

\subsubsection{Non respect de la jonction émail-cément et du système d'attache}

Une lésion du système d'attache situé au niveau de la jonction émail-cément (JEC) de la dent incluse lors du dégagement chirurgical peut avoir de dramatiques conséquences : ankylose, résorption cervicale, perte d'attache menant à la formation de poche parodontale ou de récession gingivale une fois la dent positionnée sur l'arcade. Certaines manœuvres peuvent être dites «à risque» pour le système d'attache de la dent incluse :

- un dégagement chirurgical au contact de la dent trop ambitieux et mal maîtrisé,

- une tentative de mobilisation à l'élévateur placé au niveau de la JEC pour éliminer une suspicion d'ankylose.

Ainsi, certains auteurs préconisent un dégagement osseux a minima de la couronne de la dent à tracter et une exposition coronaire raisonnée, afin d'éviter de léser son système d'attache par une intervention trop invasive de ses tissus de soutien $[4,25]$.

\subsubsection{Lésion de la dent à dégager, des dents} adjacentes et de leur tissu de soutien

Une attention toute particulière est également portée à la dent lors des manœuvres de dégagement, notamment en cas d'utilisation d'instruments rotatifs. Son environnement immédiat, tels que les dents adjacentes et leurs tissus de soutien respectifs, doit également être manipulé avec précaution. Les différentes incisions sont donc réalisées, autant que possible, à distance de leur système d'attache [24] en respectant leur gencive marginale. Le dégagement osseux est raisonné et réalisé a minima.

Lindication de la technique chirurgicale à adopter est réfléchie après étude approfondie de la situation parodontale environnant la dent retenue et de la position de celle-ci ramenée à la ligne muco-gingivale. Lobjectif étant d'aménager un contexte parodontal en quantité et en qualité satisfaisante, ceci passe par le respect de la jonction émail-cément de la dent à tracter. En effet, une atteinte de son système d'attache peut être à l'origine d'une ankylose ou d'une résorption cervicale de la racine. Lune comme l'autre peuvent stopper la dent incluse dans sa progression jusqu'à l'arcade, et mettre en jeu le pronostic de mise en place de la dent sur l'arcade.

\subsection{3. Échec de la technique de collage}

\subsubsection{Absence d'hémostase}

Certaines précautions peuvent être prises pour obtenir et conserver une bonne hémostase pendant toute la phase de collage [24] :

- réaliser des incisions de décharge à distance de la zone de trépanation osseuse,

- utiliser une solution anesthésique adrénalinée,

- éviter l'utilisation du spray d'air pour le séchage de la couronne après son mordançage.

Si, malgré cela, les conditions ne permettent pas un collage de qualité, un collage post-opératoire reste parfois possible (lambeau déplacé apicalement ou latéralement et apicalement, et lambeau palatin fenêtré) [23].

\subsubsection{Décollage de l'attache orthodontique}

Le praticien se doit de réintervenir quand il se produit un décollement du bouton d'ancrage associé à un lambeau repositionné, alors même que la dent à tracter est encore en position sous-muqueuse. Dans ce cas, le praticien doit de nouveau réaliser une intervention visant à lever un lambeau et à coller l'attache de nouveau. Une étude [5] a montré que cela se produit dans $17 \%$ des cas lorsque l'attache est collée 


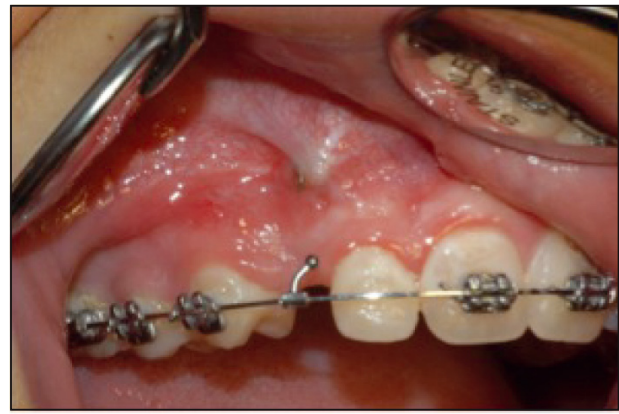

Figure 9

Vue endobuccale d'une chaînette fracturée nécessitant une réintervention chez un patient de 14 ans (document $\mathrm{N}$. Pignoly).

en per-opératoire sous un lambeau repositionné. Il semblerait de plus que les échecs de collage soient plus fréquents lorsque l'attache est collée en postopératoire [36]. Cela est cependant moins préjudiciable, car le bouton peut être recollé sans nécessité de lever un nouveau lambeau.

Une fracture de la chaînette reliant l'attache au dispositif orthodontique intra-buccal peut également se produire, de manière plus insolite. Dans le cas où la dent incluse est encore sous muqueuse, une réintervention chirurgicale est, là aussi, nécessaire (Fig. 9).

\subsubsection{Causes iatrogènes ankylose/résorption radiculaire (acide orthophosphorique, lésion radiculaire...)}

De même que des traumatismes mécaniques du système d'attache de la dent incluse peuvent avoir lieu pendant la chirurgie de dégagement, des lésions chimiques de la JEC peuvent également se produire pendant la phase de collage. Ainsi, une fusée non contrôlée d'acide orthophosphorique lors du mordançage, de l'adhésif ou de résine composite nécessaire au collage peuvent avoir de graves conséquences sur le système d'attache.

La phase de collage lors de la chirurgie de dégagement est une étape qui doit être réalisée de façon rigoureuse, avec une hémostase du site contrôlée, pour éviter les décollements intempestifs. Les matériaux du collage sont utilisés de manière raisonnée pour ne pas créer d'agression chimique du système d'attache et risquer de provoquer ankylose ou résorption radiculaire de la dent retenue.

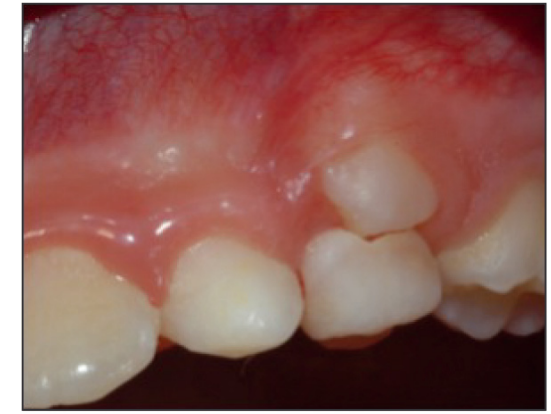

Figure 10

Persistance de la canine lactéale impliquant l'enclavement vestibulaire de la canine définitive chez une patiente de 10 ans (document N. Pignoly).

\subsection{4. Échec lié à la phase orthodontique}

\subsubsection{Prévention et interception orthodontique}

Kurol [19] souligne l'importance d'un diagnostic précoce des anomalies d'éruption pour pouvoir intervenir au moment idéal et intercepter les problèmes au moment opportun. Certains auteurs préconisent donc des contrôles radiographiques systématiques chez les patients présentant des anomalies des séquences d'éruption [1]. Souvent, ces anomalies sont en lien avec des facteurs étiologiques (dents surnuméraires, odontomes, manque d'espace disponible sur arcade..) qui, s'ils sont levés rapidement, peuvent suffire à observer une éruption physiologique de la dent retenue jusqu'à l'arcade [28] (Fig. 10).

Un lien ayant été établi entre rétention vestibulaire dans le secteur antérieur et encombrement, le praticien se doit d'éviter une fermeture d'espace par migration dentaire suite à la perte prématurée d'une dent temporaire. Si l'espace n'a pas été maintenu, il doit alors être rapidement recréé afin d'éviter un blocage du phénomène d'éruption. C'est en denture mixte que ce couloir éruptif doit être recréé, lorsque la dent retenue conserve encore un potentiel éruptif. Les différents moyens mis à sa disposition sont l'expansion, la distalisation des secteurs latéraux avec ou sans avancement du groupe incisif, ou l'avulsion des prémolaires. Cela est décidé après examen et étude céphalométriques [24].

Lavulsion de la dent temporaire permet de modifier le trajet d'éruption de la dent permanente car une dent en évolution se déplace dans le trajet de moindre résistance, et ainsi d'activer son éruption. Selon Ericson et Kurol [19], l'extraction de la canine 


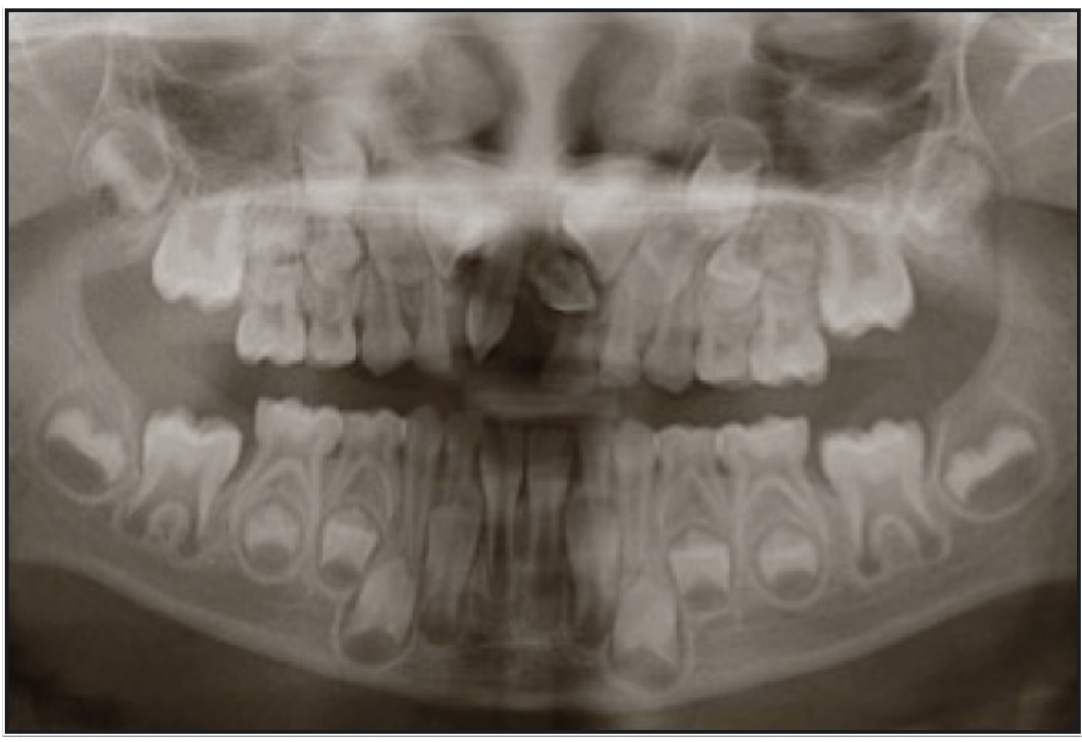

a

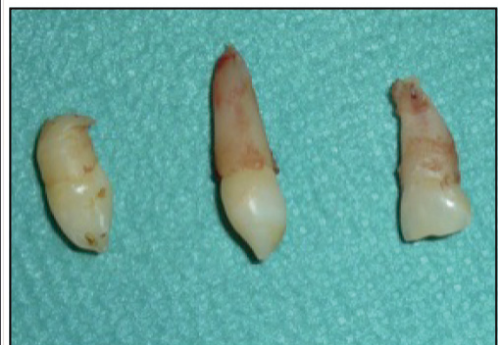

b

Figure 11

Découverte fortuite sur une radiographie panoramique de deux dents surnuméraires coniques empêchant l'éruption des incisives centrales permanentes chez un patient âgé de 7 ans.

lactéale chez des enfants âgés de 10 à 13 ans et présentant une faible perte d'espace permet la mise en place spontanée dans $78 \%$ des cas. Mais cela semble dépendre de la position de la canine permanente. Pour les incisives centrales maxillaires, seulement $50 \%$ de ces dents ont spontanément fait leur éruption sans aide chirurgicale ni orthodontique, après la levée des obstacles.

De même, en cas de présence d'obstacles à l'éruption d'une dent définitive dans le secteur antérieur, leur avulsion doit être rapidement programmée (Fig. 11). En effet, si une expansion maxillaire rapide concomittente est réalisée, une éruption spontanée de la définitive s'effectue alors dans $82 \%$ des cas en six à sept mois. Sans cette expansion maxillaire, l'éruption se fait tout de même dans 39 \% des cas, malgré un manque d'espace dans la région antérieure [33].

La création ou le maintien d'un espace disponible sur la crête associé à l'avulsion de la canine lactéale ou d'obstacles à l'éruption type odontome ou dent surnuméraire peuvent suffire à réactiver une éruption physiologique d'une canine retenue si cette interception a lieu avant 13 ans.

\subsubsection{Immobilité de la dent malgré les tractions orthodontiques}

Il peut arriver que, malgré une thérapeutique chirurgico-orthodontique mise en place avec rigueur, aucun mouvement de la dent incluse ne soit observé après activation de la traction orthodontique.

Bien que l'imagerie 3D permette de mieux visualiser la position et l'orientation de la dent, il reste délicat pour l'orthodontiste de gérer la direction des forces à appliquer sur une dent incluse. C'est d'autant plus le cas lorsqu'un lambeau est repositionné et ne permet pas à l'orthodontiste de voir la position de la couronne au fil des séances. La traction se fait donc « à l'aveugle » et peut engendrer des erreurs de direction ayant des conséquences sur les suites du traitement.

Trop souvent, le phénomène d'ankylose est avancé comme cause d'immobilité de la dent incluse face à la traction exercée. Cependant, il semblerait que des erreurs de traction, en intensité et en direction, sont plus souvent responsables d'une absence de mouvement de la dent incluse, bien plus que le phénomène d'ankylose, d'ailleurs extrêmement rare chez les patients jeunes [4].

Une immobilité de la dent incluse pendant sa traction orthodontique résulte le plus souvent d'un 


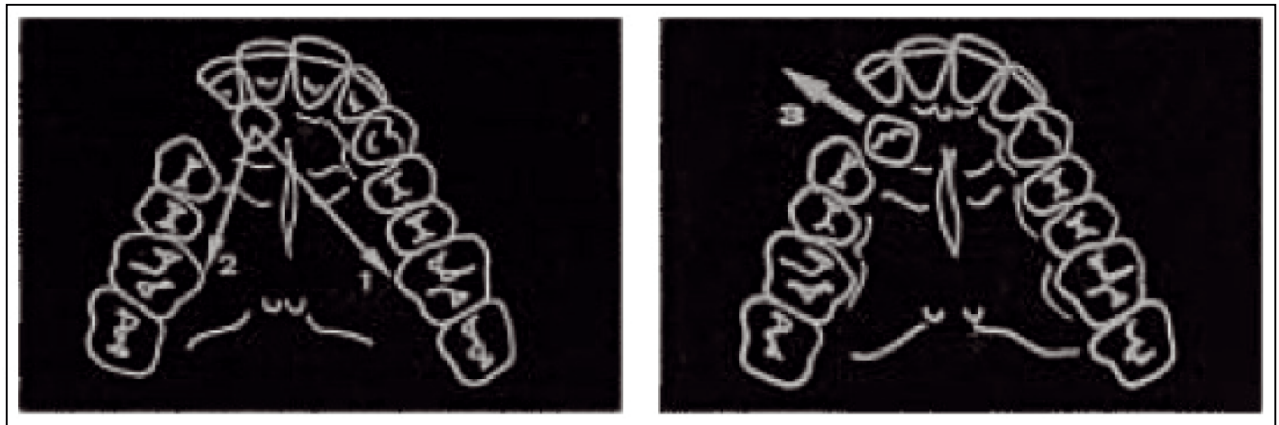

Figure 12

Direction des forces de tractions orthodontiques qui permet de réduire les risques de problèmes parodontaux (d'après Crinetz [15]).

vecteur de force non adapté, ou plus rarement d'un phénomène d'ankylose ou encore d'un décollement de l'attache orthodontique.

\subsubsection{Non respect des structures anatomiques par des axes/forces de traction inadaptés}

Lorsque la dent incluse est une canine, quelque soit sa localisation vestibulaire ou palatine, le chemin le plus court menant à sa place finale sur l'arcade passe souvent par la racine de l'incisive latérale, voire de l'incisive centrale. Dans les cas simples, une petite interférence peut être surmontée en augmentant la force de traction mais, si l'ampleur de l'interférence est plus importante, cela peut ne pas suffire à tracter la dent incluse. Augmenter encore davantage les forces augmente la charge sur la dent et notamment sur son moyen d'ancrage qui risque le décollement, sans parler des risques de résorptions des racines incisives : création de résorptions ou aggravation de résorptions existantes.

Lorsque la canine est en position palatine, et afin d'éviter ce risque potentiel d'échec, la traction est divisée en deux étapes. La première consiste à éloigner la canine incluse des racines incisives jusqu'à une position où sa trajectoire directe jusqu'à sa position finale sur l'arcade n'interfère plus avec les racines des dents adjacentes. Le déplacement se fait en position légèrement plus palatine encore, mais déjà en direction distale jusqu'à sa position finale. Une fois éloignée des racines adjacentes, la dent peut alors être tractée en direction plus vestibulaire par un simple mouvement de bascule (tipping) jusqu'à l'arc vestibulaire, évitant ainsi les échecs liés à la fois aux pertes d'ancrage et aux résorptions potentielles des dents adjacentes (Fig. 12).
Lorsque la canine est incluse en position vestibulaire, l'orthodontiste doit éviter de la tracter en direction vestibulaire. Cela induirait une déhiscence osseuse par résorption de la corticale vestibulaire avec comme conséquence finale une récession gingivale associée. Les forces de traction doivent donc s'orienter verticalement, ayant pour objectif de faire émerger la canine le plus proche possible du sommet de la crête alvéolaire [14].

Concernant les forces de traction, elles doivent être légères et continues pour éviter des résorptions osseuses et favoriser une bonne intégration gingivale en permettant au parodonte superficiel préparé par lambeau de suivre la dent lors de sa mise en place sur l'arcade [25]. La force conseillée pour une canine maxillaire est de l'ordre de $30 \mathrm{~g}$ pour le mouvement de déplacement vertical (extrusion) et de $50 \mathrm{~g}$ pour le déplacement vestibulaire [24]. En cas d'extrusion rapide de la dent incluse par des forces importantes, les tissus parodontaux peuvent avoir du mal à suivre. En résultent des récessions gingivales inesthétiques associées à des déhiscences osseuses sous jacentes.

D'autres complications peuvent aboutir à l'utilisation de forces orthodontiques trop lourdes : un phénomène d'ankylose dû à un étirement trop important et rapide du ligament desmodontal, ou encore des phénomènes de résorptions externes radiculaires. Ainsi, les forces optimales peuvent varier aux alentours de $15 \mathrm{~g}$ pour une incisive. Une traction physiologique aboutit à une extrusion d'un millimètre par semaine. Après la phase de traction, une phase de stabilisation de six à huit semaines doit être respectée pour une maturation des tissus parodontaux déplacés. Enfin, une contention de six mois 
minimum achève de maintenir la dent dans sa position définitive et évite une récidive partielle [35].

Il est préférable de laisser les dents adjacentes «libres » de tout mouvement. C'est notamment le cas de l'incisive latérale lors de la traction d'une canine incluse en position palatine. Cela évite des dommages radiculaires en cas de forte proximité entre la canine et la racine de l'incisive latérale lors de la traction.

Les forces de traction orthodontiques, légères et continues, doivent être associées à des directions permettant à la dent retenue d'éviter un rapport trop intime avec les structures environnantes pouvant être à l'origine de résorptions radiculaires.

\subsubsection{Erreur du choix d'appareil}

Il existe différents types d'ancrage, plus ou moins respectueux de la dent incluse, des dents adjacentes et du parodonte environnant $[16,17,36,39]$.

Les premiers ancrages étaient intra-coronaires. Qu'ils soient scellés ou vissés, ils ont rapidement été abandonnés du fait des nécroses pulpaires et de la mutilation systématique de la couronne qui nécessitait une restauration une fois la dent mise en place sur l'arcade.

Les ancrages ont ensuite été péri-coronaires. La ligature métallique au collet, plus communément appelée la «technique du lasso », a été largement utilisée. Placée au contact de la jonction émail-cément, elle engendrait des lyses osseuses lors de son déplacement, des résorptions externes, des phénomènes d'ankylose ainsi qu'une parodontite expérimentale. Les bagues ou les coiffes scellées nécessitaient, de même que la technique du lasso, un dégagement osseux important de la couronne pour leur mise en place, évalué à plus d'l mm dans une étude menée par Boyd [10]. Cela entraînait des pertes osseuses supplémentaires et des risques de lésions des éléments dentaires environnants.

Désormais, les attaches collées permettent d'être respectueuses, à la fois des tissus dentaires et parodontaux. Leur petite taille permet un dégagement limité permettant de préserver le sac folliculaire ainsi que la jonction émail-cément.

\subsection{5. Échec lié à la prise en charge multidisciplinaire}

La phase chirurgicale de désinclusion se décide en étroite collaboration entre le chirurgien-dentiste qui réalise l'acte de dégagement et l'orthodontiste qui met en place les dispositifs de traction.

\subsubsection{Mauvaise communication «ortho-paro »}

Une intervention chirurgicale non précédée d'un aménagement de l'espace d'émergence, ni suivi d'une thérapeutique orthodontique reste, même si elle est parfaitement réalisée, inutile et vaine.

Le site receveur doit être aménagé avec un excès de place de $2 \mathrm{~mm}$ car la dent réalise souvent une rotation dans l'espace, ce qui augmente l'espace nécessaire à ses mouvements [5]. Différents moyens sont mis à la disposition de l'orthodontiste pour recréer cet espace disponible : fermeture de diastèmes incisifs, recul du secteur latéral, avancement du groupe incisif, augmentation du périmètre d'arcade et/ou avulsion de dent(s) permanente(s) [24]. Laménagement de l'espace permet également de valider la faisabilité du traitement et d'éviter des phénomènes de résorption par des mouvements trop précoces d'une dent incluse dont le couloir éruptif n'a pas été préparé.

Il est indispensable qu'un espace suffisant soit créé ou maintenu sur l'arcade avant que la thérapeutique chirurgico-orthodontique ne soit mise en place.

\subsection{6. Échec lié au facteur temps}

\subsubsection{Abstention}

La découverte d'une dent incluse reste le plus souvent fortuite, lors d'un examen de contrôle. De nombreux examens radiologiques sont réalisés. Tout un arsenal thérapeutique est ensuite proposé au patient, comprenant le plus souvent un traitement orthodontique pré-opératoire et/ou post-opératoire.

Le patient qui apprend alors avec surprise le diagnostic de l'inclusion peut réagir de différentes manières :

- Lincompréhension face à des thérapeutiques de grande ampleur dont il ne ressent pas le besoin.

- Lanxiété concernant les phases chirurgicales.

- Le manque de motivation et l'incapacité financière face à un traitement long et coûteux impliquant des rendez-vous réguliers et nécessitant une participation rigoureuse.

Cela concerne davantage l'adulte chez lequel la découverte de l'inclusion est tardive, fortuite et sans symptomatologie associée. 


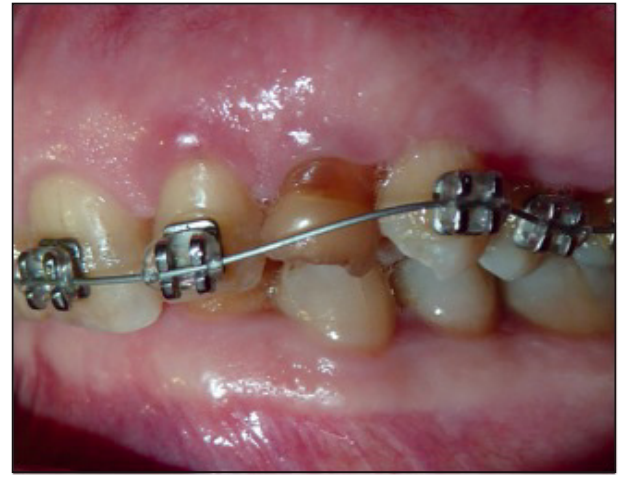

Figure 13

Canine maxillaire lactéale chez une patiente de 52 ans présentant une abrasion sévère ainsi qu'une dyschromie.

Chez l'enfant, les choses sont différentes. Les parents souhaitant le meilleur pour leur enfant, que ce soit sur le plan fonctionnel ou esthétique, ils s'engagent avec moins de difficulté dans ce type de thérapeutique multi-disciplinaire.

En cas d'abstention, la dent incluse est surveillée à la fois cliniquement et radiographiquement tous les 18 à 24 mois [20] afin d'intercepter toute pathologie évolutive de la dent laissée en place.

Si la dent lactéale est maintenue sur l'arcade, il faut prévenir le patient de l'incertitude de son pronostic à long terme, et de la nécessité d'un contrôle régulier et rigoureux. Sa faible longueur radiculaire, associée au risque carieux ou de rhizalyse, joue un rôle certain dans la mobilité de cette dent déjà soumise aux mouvements répétés de diduction. Au fil des années, son abrasion réduit d'autant plus son rapport de taille avec les dents permanentes adjacentes et peut devenir un critère inesthétique non négligeable pour le patient (Fig. 13).

Sa perte, ou la décision du patient de la faire extraire, engendre alors des difficultés de remplacement, tant sur le point fonctionnel qu'esthétique.

L'abstention, bien qu'elle ne doive pas être écartée compte tenu du rapport bénéfice-risque d'une intervention sur une dent retenue, doit être considérée comme une réelle solution de compromis. Elle nécessite une surveillance rigoureuse et sur le long terme de la dent lactéale sur l'arcade et de la dent incluse laissée en place.

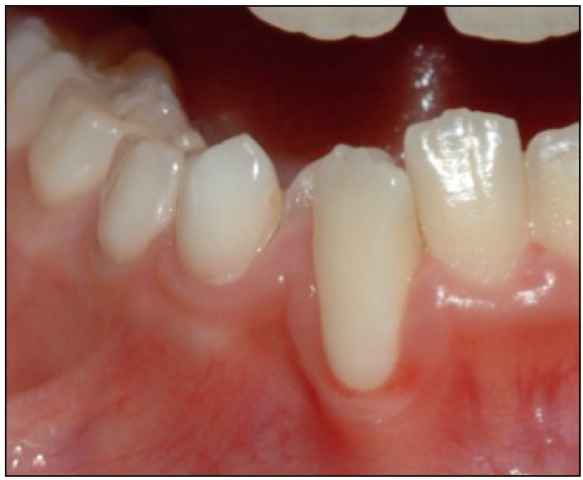

Figure 14

Canine mandibulaire en position ectopique vestibulaire ayant émergé dans un espace dépourvu de gencive kératinisée chez une patiente de 10 ans (document N. Pignoly).

\subsubsection{Durée de la temporisation}

\subsection{Quand intervenir?}

Le traitement est initié de préférence lorsque le développement radiculaire est compris entre la moitié et les $2 / 3$ de sa longueur. Il serait inapproprié d'exposer une dent dont la formation radiculaire est insuffisante. À ce stade, la dent ne peut pas être considérée comme incluse. Il faut alors commencer par lever les obstacles à l'éruption et laisser la dent se servir de son pouvoir éruptif élevé pour continuer son éruption. Agir trop précocement peut endommager la couronne et avoir des conséquences néfastes sur le développement radiculaire [2].

\subsubsection{2. Éruption en muqueuse alvéolaire}

Le point d'émergence d'une dent permanente doit se faire sous surveillance. En effet, elle peut aboutir à une éruption en muqueuse alvéolaire, sans que les tissus parodontaux n'aient été aménagés (Fig. 14).

Une récession gingivale peut alors être observée en cas de temporisation trop longue, de non suivi de l'éruption ou d'absence d'intervention au bon moment pour un aménagement parodontal optimisé (Fig. 15).

Dans le cas d'une canine en position ectopique dont l'éruption risque de se faire en muqueuse alvéolaire, la décision est de prévoir l'acte chirurgical avant l'émergence afin de pouvoir adapter la technique de dégagement à une conservation ou une augmentation du parodonte environnant. 


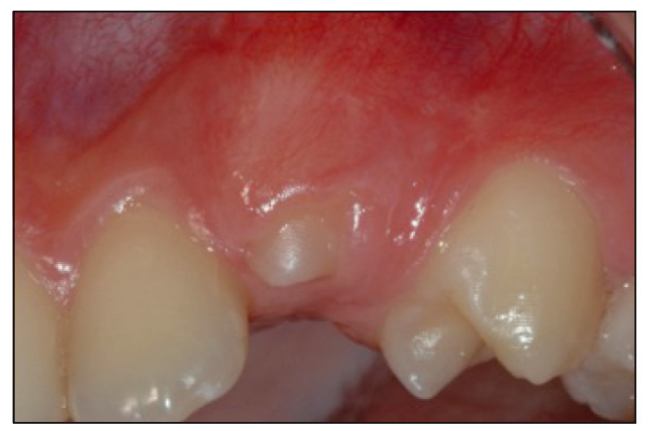

Figure 15

Éruption de la 23 dans un milieu faiblement pourvu de gencive kératinisé chez un patient de 12 ans (document $\mathrm{N}$. Pignoly).

C'est également le cas d'une dent retenue en position haute ou trans-alvéolaire en cours de traction après réalisation d'un lambeau replacé. Une fois la dent arrivée au niveau de la ligne muco-gingivale, une nouvelle intervention par lambeau déplacé cette fois doit avoir lieu s'il existe un risque d'émergence en muqueuse alvéolaire.

\subsubsection{Résorption interne de la dent mise en place}

Les manœuvres effectuées lors de la chirurgie de dégagement, ou plus tardivement lors des mouvements de traction orthodontique, peuvent entraîner une inflammation pulpaire chronique et irréversible; c'est le phénomène de résorption interne. Les signes cliniques ne se déclarent que tardivement, par une fracture coronaire et/ou radiculaire ainsi qu'une coloration rosée de la couronne. Du fait de ces manifestations tardives, des contrôles radiologiques réguliers des dents incluses mises en place sur l'arcade doivent être programmés.

\subsection{7. Échec lié à la dent/au patient}

\subsubsection{Morphologie}

En cas de traumatisme survenu pendant la petite enfance, le germe en formation peut aboutir soit à une dent dilacérée, soit à une dent dite "angulée» (Fig. 16). Alors que l'indication d'avulsion est posée pour une dent dilacérée, le dégagement chirurgicoorthodontique peut être tenté pour une dent angulée, avec toutefois un risque notable de fenestration de la corticale vestibulaire du fait de la coudure radiculaire. L'environnement parodontal doit alors être

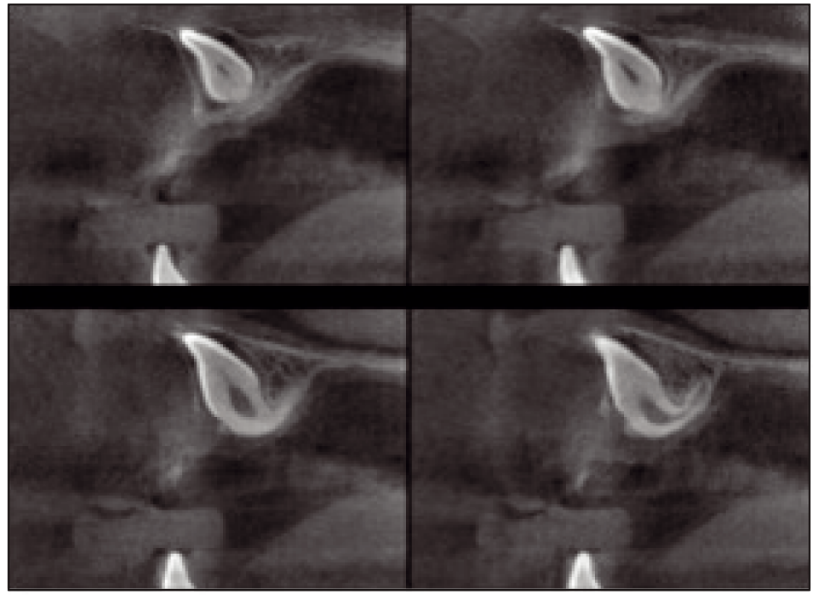

Figure 16

Images tomodensitométriques d'une dent angulée (document V. Monnet-Corti).

évalué, car une fenestration met en jeu le pronostic de la dent à moyen voire court terme.

Dans le cas des dents angulées, il faut veiller à bien évaluer le risque de fenestration de la corticale vestibulaire et porter une attention toute particulière au fort taux d'ankylose concernant ces dents traumatisées.

En cas de possible mise en place de la dent angulée sur l'arcade, deux chirurgies sont habituellement nécessaires: tout d'abord un lambeau repositionné permettant de coller la première attache; puis c'est un lambeau déplacé apicalement qui est réalisé lorsque le bord libre s'apprête à faire son éruption en muqueuse alvéolaire, afin d'aménager son éruption en gencive kératinisée [11] (Fig. 17). Cependant, du fait de sa localisation et de son angulation, un dégagement en muqueuse alvéolaire est parfois inévitable pour permettre une traction convenable et efficace. Un aménagement par un lambeau positionné coronairement doit rapidement être réalisé afin de recréer un bandeau suffisant de tissu kératinisé, indispensable à une architecture gingivale satisfaisante en fin de traitement [34] (Fig. 18).

Avec près de 20 \% d'échec de mise en place d'une dent angulée sur l'arcade. Becker, et al. estiment que ce fort taux d'échec, plus qu'à son anatomie radiculaire, est surtout lié au fait que la dent angulée présente fréquemment des problèmes d'ankylose et de résorption [11]. 


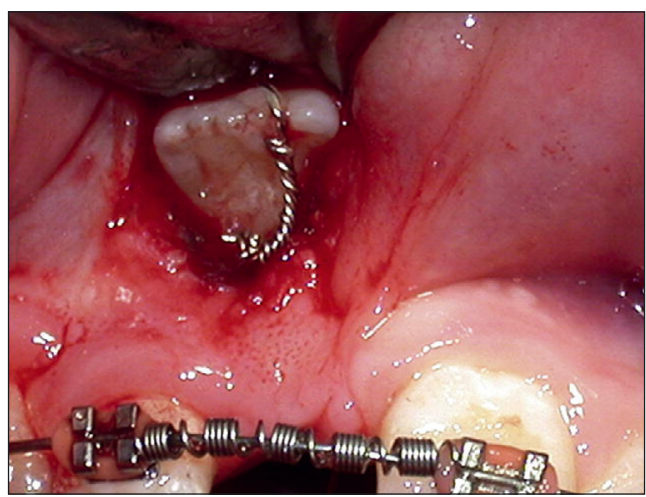

a

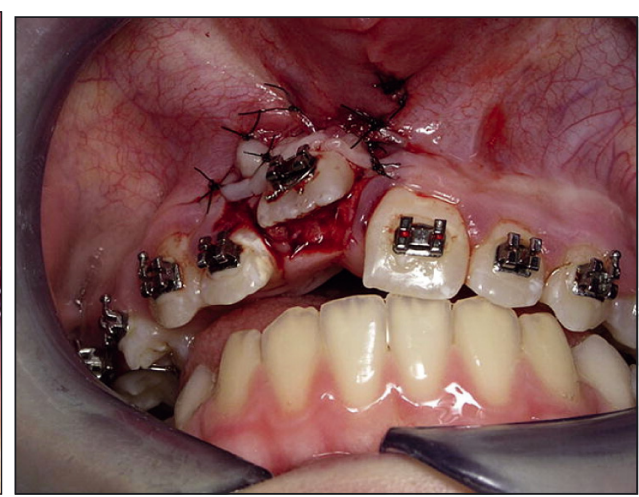

b

Figure 17

Dégagement de la 11 incluse en position ectopique vestibulaire et aménagement rapide des tissus parodontaux grâce à un lambeau positionné apicalement (d'après Pinho, et al. [34]).

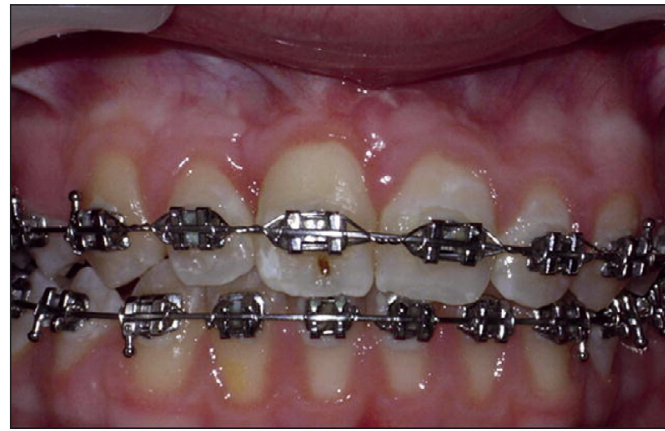

Figure 18

Situation finale en fin de traitement présentant une architecture gingivale satisfaisante (d'après Pinho, et al. [34]).

\subsubsection{2. Âge}

Lâge est un facteur pouvant influencer la durée ainsi que le pronostic de la thérapeutique chirurgico-orthodontique. Ainsi, il semblerait que les patients âgés de plus de 40 ans auraient moins de chance de voir aboutir avec succès leur traitement. En effet, plus le patient avance en âge, et plus les chances de succès s'amenuisent, notamment à cause du risque d'ankylose [4]. Dans une étude menée par Becker, le taux de réussite des traitements chirurgico-orthodontiques chez l'enfant ont été comparés aux taux de réussite chez l'adulte. Alors que chez l'enfant, le taux était de $100 \%$, pour l'adulte le taux s'élevait à seulement 69,5\% sur une vingtaine de dents traitées [3].

\subsubsection{Localisation - angulation}

Certains facteurs radiologiques prédisent des difficultés de traitement, et orientent davantage

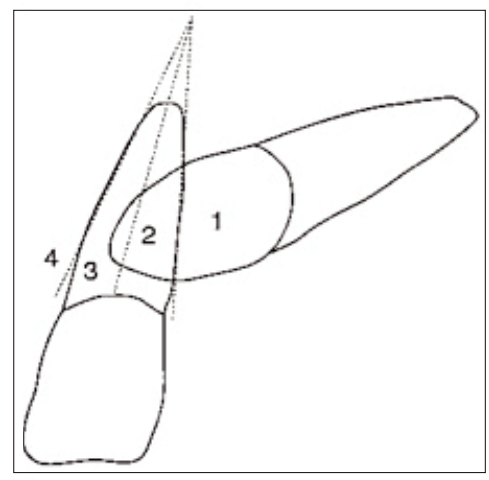

Figure 19

Stade de superposition de la canine incluse sur la racine de l'incisive latérale (d'après Stivaros, et al. [38]).

le traitement vers une extraction de la dent incluse plutôt qu'une thérapeutique chirurgicoorthodontique [31] :

- Un grade 3-4 de superposition de la canine incluse sur l'incisive latérale (Fig. 19).

- Un angle entre le grand axe de la dent incluse et la ligne médiane supérieur à $45^{\circ}$ (Fig. 20).

Alors que la position plus ou moins horizontale d'une canine retenue, ainsi que sa superposition au niveau des racines des dents adjacentes peuvent prédire des difficultés et des échecs de la mise en place, d'autres facteurs comme la hauteur d'inclusion semblent ne pas intervenir dans ce pronostic de mise en place. 

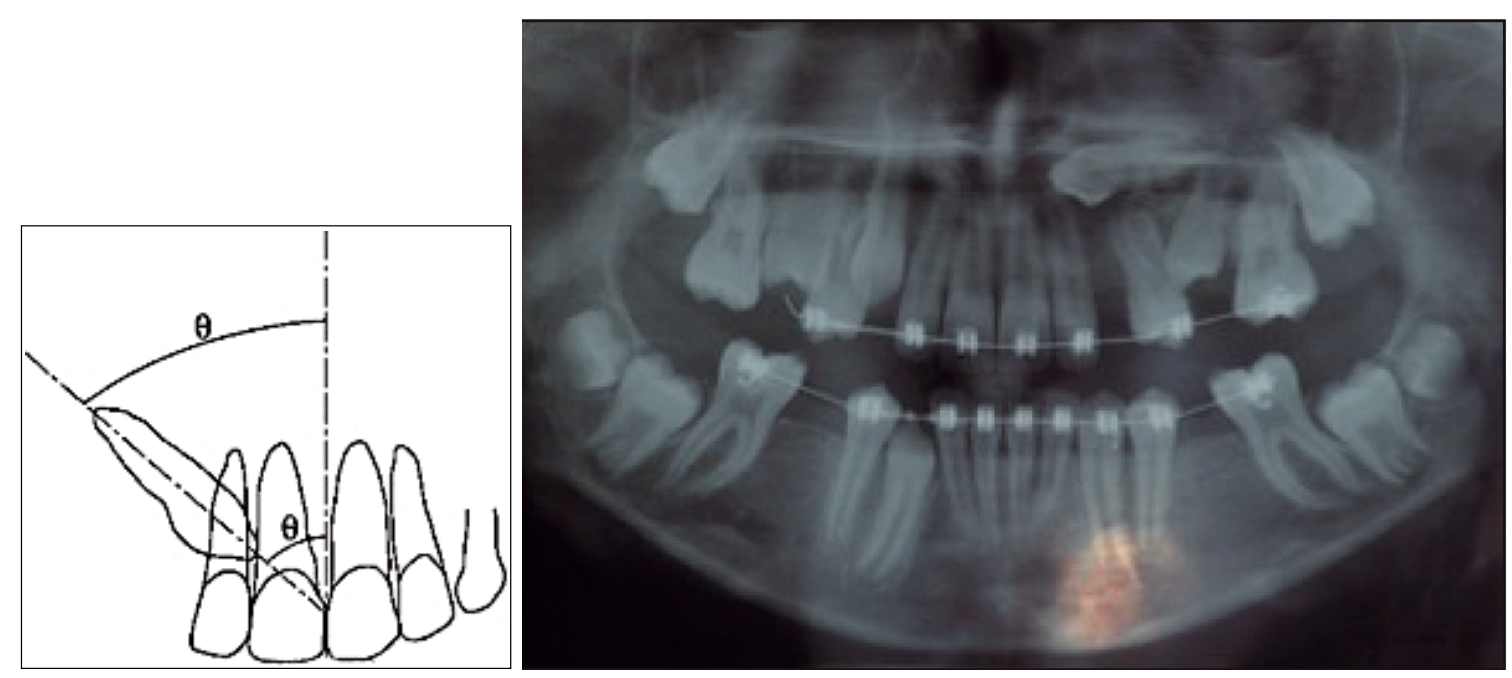

b

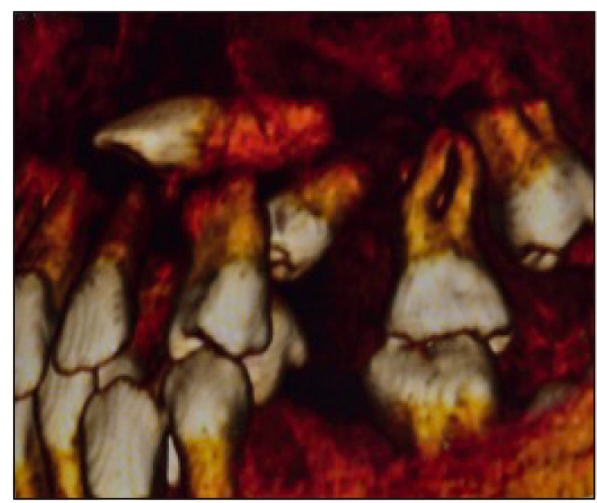

c

Figure 20

Angle entre l'axe de la canine incluse et la ligne médiane, et exemple clinique (d'après Stivaros, et al. [38]).

\subsubsection{Ankylose - Résorption}

La dent incluse peut présenter, sur les examens radiologiques, un phénomène de résorption qui met en jeu la possibilité de sa mise en place chirurgicoorthodontique sur l'arcade, et son pronostic à court ou moyen terme.

De même, l'ankylose peut empêcher la mise en place de la dent incluse jusqu'à sa position définitive sur l'arcade. Qu'elle soit diagnostiquée précocément ou après des tentatives répétées de traction, cette fusion du cément et de l'os avec oblitération de l'espace desmodontal bloque toute évolution dentaire physiologique ou provoquée.

\section{Conflit d'intérêts}

Les auteurs déclarent n'avoir aucun lien d'intérêt concernant les données publiées dans cet article.

\section{Bibliographie}

[1] Batra P, Duggal R, Kharbanda OP, Parkash H. Orthodontic treatment of impacted anterior teeth due to odontomas: a report of two cases. J Clin Pediatr Dent 2004;28:289-294.

[2] Becker A. The orthodontic treatment of impacted teeth. Thieme; 2007.

[3] Becker A, Chaushu S. Success rate and duration of orthodontic treatment for adult patients with palatally impacted maxillary canines. Am J Orthod Dentofacial Orthop 2003;124:509-514.

[4] Becker A, Chaushu G, Chaushu S. Analysis of failure in the treatment of impacted maxillary canines. Am J Orthod Dentofacial Orthop 2010;137:743-754.

[5] Becker A, Shpack N, Shteyer A. Attachment bonding to impacted teeth at the time of surgical exposure. Eur J Orthod 1996;18:457-463.

[6] Bishara SE, Ortho. D. Impacted maxillary canines: A review. Am J Orthod Dentofacial Orthop 1992;101:159_ 171.

[7] Boileau M-J. Orthodontie de l'enfant et du jeune adulte: Principes et moyens therapeutiques. Elsevier Masson; 2011, 1039 p. 
[8] Boisramé-Gastrin S, Denhez F. Extractions des canines et autres dents incluses. Encycl Med Chir, Odontologie, Paris: Elsevier 2010, 28-755-Q-10.

[9] Borghetti A, Monnet-Corti V. Chirurgie plastique parodontale. Editions CDP; 2008.

[10] Boyd RL. Clinical assessment of injuries in orthodontic movement of impacted teeth: I. Methods of attachment. Am J Orthod 1982;82:478-486.

[11] Chaushu S, Becker T, Becker A. Impacted central incisors: Factors affecting prognosis and treatment duration. Am J Orthod Dentofacial Orthop 2015;147:355-362.

[12] Chen Y, Duan P, Meng Y, Chen Y. Three-dimensional spiral computed tomographic imaging: a new approach to the diagnosis and treatment planning of impacted teeth. Am J Orthod Dentofacial Orthop 2006;130:112-116.

[13] Crescini A, Nieri M, Buti J, Baccetti T, Mauro S, Prato GPP. Short- and long-term periodontal evaluation of impacted canines treated with a closed surgical-orthodontic approach. J Clin Periodontol 2007;34:232-242.

[14] Crescini A, Nieri M, Buti J, Baccetti T, Pini Prato GP. Pre-treatment radiographic features for the periodontal prognosis of treated impacted canines. J Clin Periodontol 2007;34:581-587.

[15] Crinetz V. Conduite à tenir devant une canine incluse en situation palatine. Rev Stomatol Chir maxillofac 1999;100:257-264.

[16] Crismani AG, Freudenthaler JW, Weber R. Canines supérieures incluses-méthodes conventionnelles de diagnostic radiologique et de traitement. Rev Mens Suisse Odontostomatol 2000;110:1264-1267.

[17] Delsol L, Orti V, Chouvin M, Canal P. Canines et incisives maxillaires incluses: diagnostic et thérapeutique. Elsevier Masson SAS; 2008.

[18] Ericson S, Kurol J. Radiographic examination of ectopically erupting maxillary canines. Am J Orthod Dentofacial Orthop 1987;91:483-492.

[19] Ericson S, Kurol J. Early treatment of palatally erupting maxillary canines by extraction of the primary canines. Eur J Orthod 1988;10:283-295.

[20] Franck CA, Long M. Periodontal concerns associated with the orthodontic treatment of impacted teeth. Am J Orthod Dentofacial Orthop 2002;121:639-649.

[21] Harzer W, Seifert D, Mahdi Y. The orthodontic classification of impacted canines with special reference to the age at treatment, the angulation and dynamic occlusion. Fortschritte Kieferorthopädie 1994;55:47.

[22] Kokich VG. Surgical and orthodontic management of impacted maxillary canines. Am J Orthod Dentofacial Orthop $2004 ; 126: 278-283$.

[23] Korbendau J-M, Guyomard. Chirurgie parodontale orthodontique. Editions CDP; 1998.

[24] Korbendau J-M, Patti A. Le Traitement orthodontique et chirurgical des dents incluses. Quintessence international; 2005, $135 \mathrm{p}$.

[25] Lallam-Laroye C, Dridi S-M, Blanc A, Colombier M-L. La désinclusion des canines maxillaires retenues: une chirurgie parodontale orthodontique essentielle. Actual Odonto-Stomatol 2008;(244):345-354
[26] Litsas G, Acar A. A review of early displaced maxillary canines: etiology, diagnosis and interceptive treatment. Open Dent J 2011;5:39-47.

[27] Liu D, Zhang W, Zhang Z, Wu Y, Ma X. Localization of impacted maxillary canines and observation of adjacent incisor resorption with cone-beam computed tomography. Oral Surg Oral Med Oral Pathol Oral Radiol Endod 2008;105:91-98.

[28] Machado AW, Maia LGM, Vianna AP, Gandini Jùnior LG. Orthodontic traction of impacted upper central incisors related to mesiodens. Rev Gaúcha Odontol 2015;63:75_ 80.

[29] Messaoudi Y, Coudert J-L, Aknin J-J. Apport de la reconstruction tridimensionnelle à l'aide du logiciel 3DNEO ${ }^{\circledR}$ dans le traitement chirurgico-orthodontique des dents incluses. Orthod Fr 2013;84:147-155.

[30] Miresmaeili A, Farhadian N, Mollabashi V, Yousefi F. Webbased evaluation of experts' opinions on impacted maxillary canines forced eruption using CBCT. Dent Press J Orthod 2015;20:90-99.

[31] Motamedi MHK, Tabatabaie FA, Navi F, Shafeie HA, Fard BK, Hayati Z. Assessment of radiographic factors affecting surgical exposure and orthodontic alignment of impacted canines of the palate: A 15-year retrospective study. Oral Surg Oral Med Oral Pathol Oral Radiol Endod 2009; 107:772-775.

[32] Paris M, Trunde F, Bossard D, Farges JC, Coudert JL. Lankylose dentaire: diagnostic par tomodensitométrie et reconstruction tridimensionnelle. J Radiol 2010;91:707711.

[33] Pavoni C, Franchi L, Lagana G, Baccetti T, Cozza P. Management of impacted incisors following surgery to remove obstacles to eruption: a prospective clinical trial. Pediatr Dent 2013;35:364-368.

[34] Pinho T, Neves M, Alves C. Impacted maxillary central incisor: Surgical exposure and orthodontic treatment. Am J Orthod Dentofacial Orthop 2011;140:256-265.

[35] Rao A, Philip P. Orthodontic extrusion of an impacted tooth with a removable appliance and a bonded attachment: A case report with relevant biomechanics. J Interdiscip Dent 2014;4:46.

[36] Sajnani AK, King NM. Retrospective audit of management techniques for treating impacted maxillary canines in children and adolescents over a 27-year period. J Oral Maxillofac Surg 2011;69:2494-2499.

[37] Samir EB. Clinical management of impacted maxillary canines. Semin Orthod 1998;4:87-98.

[38] Stivaros N, Mandall NA. Radiographic factors affecting the management of impacted upper permanent canines. J Orthod 2000;27:169-173.

[39] Thomine F, Korbendau J, Martineau C. Mise en place chirurgico-orthodontique des dents retenues. Real Clin 1995;6:351-358.

[40] Thoné M, Reychler H. Auto-transplantation de canine maxillaire incluse ou retenue. Rev Stomatol Chir Maxillofac 2002;5(103):288-293. 